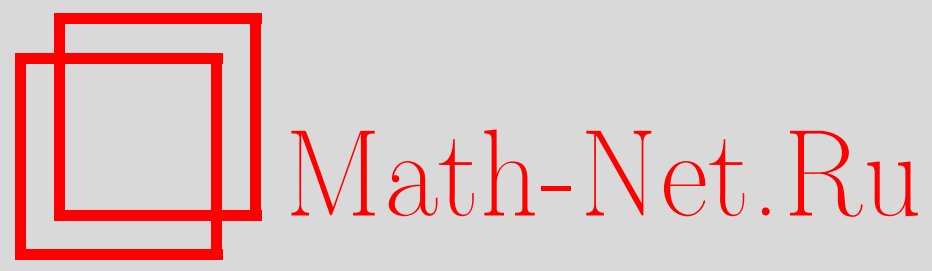

Н. И. Казимиров, Возникновение гигантской компоненты в случайной подстановке с известным числом циклов, Дискрет. матем., 2003, том 15, выпуск 3, 145-159

DOI: https://doi.org/10.4213/dm212

Использование Общероссийского математического портала Math-Net.Ru подразумевает, что вы прочитали и согласны с пользовательским соглашением http://www mathnet.ru/rus/agreement

Параметры загрузки:

IP: 44.207 .124 .84

26 апреля 2023 г., 12:33:47

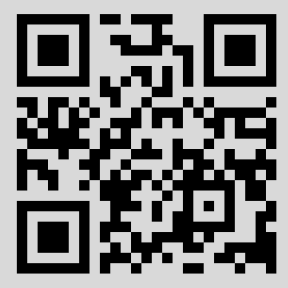




\title{
Возникновение гигантской компоненты в случайной подстановке с известным числом циклов
}

\author{
() 2003 г. Н. И. Казимиров
}

\begin{abstract}
Найдены условия возникновения гигантского цикла в случайной подстановке с известным числом циклов, получены предельные распределения наибольших длин циклов во всех зонах изменения параметров.
\end{abstract}

В [1] изучен ряд свойств случайной подстановки множества $X_{n}=\{1, \ldots, n\}$, которая совпадает с любым взаимно однозначным отображением $X_{n}$ на себя с вероятностью $(n !)^{-1}$. В этой статье рассмотрено предельное поведение наибольших длин циклов случайной подстановки с известным числом циклов. На основании этих результатов найдены условия возникновения гигантского цикла в такой случайной подстановке.

Пусть $\pi_{N . n}-$ случайная подстановка множества $X_{n}$, содержащая ровно $N$ циклов и равномерно распределенная на множестве всех подстановок $X_{n}$ с $N$ циклами (понятно, что $n \geqslant N)$. Обозначим $\eta_{1}, \ldots, \eta_{N}$ длины циклов случайной подстановки $\pi_{N . n}$, занумерованных одним из $N$ ! возможных способов. Тогда, как показано в [1], для любых целых чисел $k_{1}, \ldots k_{N}$ имеет место соотношение

$$
\mathbf{P}\left\{\eta_{1}=k_{1}, \ldots, \eta_{N}=k_{N}\right\}=\mathbf{P}\left\{\xi_{1}=k_{1}, \ldots, \xi_{N}=k_{N} \mid \xi_{1}+\cdots+\xi_{N}=n\right\},
$$

где случайные величины $\xi_{1}, \ldots, \xi_{N}$ независимы и имеют распределение логарифмического ряда, то есть

$$
\mathbf{P}\left\{\xi_{1}=k\right\}=\frac{x^{k}}{k \ln (1-x)^{-1}}, \quad 0<x<1, \quad k=1,2, \ldots
$$

Таким образом, для получения предельных распределений длин циклов случайной подстановки $\pi_{N . n}$ можно использовать обобщенную схему размешения [1-3].

В [4] доказано, что с помощью этой же обобщенной схемы размещения можно изучать предельное поведение объемов деревьев в рекурсивном лесе, состоящем из $N$ некорневых деревьев и содержашем $n$ вершин, при равномерном распределении вероятностей на множестве таких лесов. В [4] получено полное описание предельного поведения максимального объема дерева, а значит, и максимальной длины цикла в случайной подстановке $\pi_{N, n}$. Ниже будет получено полное описание предельного поведения $p$-й по величине компоненты случайной подстановки $\pi_{N . n}$ для любого фиксированного $p$. Поскольку, 
как отмечалось выше, для изучения рекурсивных лесов также используется обобщенная схема размещения (1), все полученные здесь результаты остаются справедливыми для случайных рекурсивных лесов.

Пусть

$$
\eta_{(1)} \leqslant \eta_{(2)} \leqslant \ldots \leqslant \eta_{(N)}
$$

- вариационный ряд случайных величин $\eta_{1}, \ldots, \eta_{N}$. Для целого $r \geqslant 1$ положим

$$
\begin{aligned}
& \mathbf{P}\left\{\xi_{j}^{(r)}=k\right\}=\mathbf{P}\left\{\xi_{1}=k \mid \xi_{1} \leqslant r\right\}, \\
& \mathbf{P}\left\{\tilde{\xi}_{j}^{(r)}=k\right\}=\mathbf{P}\left\{\xi_{1}=k \mid \xi_{1}>r\right\}, \quad j=1, \ldots, N,
\end{aligned}
$$

при этом случайные величины $\xi_{1}^{(r)}, \ldots, \xi_{N}^{(r)}, \tilde{\xi}_{1}^{(r)}, \ldots, \tilde{\xi}_{N}^{(r)}$ независимы. Пусть также

$$
\begin{aligned}
\zeta_{N} & =\xi_{1}+\ldots+\xi_{N}, \\
\tilde{\zeta}_{N}^{(r)} & =\xi_{1}^{(r)}+\ldots+\xi_{N}^{(r)}, \\
\tilde{\zeta}_{0}^{(r)} & =0 .
\end{aligned}
$$

В [1] доказано равенство

$$
\mathbf{P}\left\{\eta_{(N-p)} \leqslant r\right\}=\sum_{l=0}^{p}\left(\begin{array}{c}
N \\
l
\end{array}\right) P_{r}^{l}\left(1-P_{r}\right)^{N-l} \frac{\mathbf{P}\left\{\zeta_{N-l}^{(r)}+\tilde{\zeta}_{l}^{(r)}=n\right\}}{\mathbf{P}\left\{\zeta_{N}=n\right\}},
$$

где $p=0,1, \ldots, N-1, P_{r}=\mathbf{P}\left\{\xi_{1}>r\right\}$. Отсюда видно, что для получения предельных распределений случайных величин $\eta_{(N-p)}$ достаточно найти предельные распределения сумм $\zeta_{N}, \zeta_{N-l}^{(r)}+\tilde{\zeta}_{l}^{(r)}$ и асимптотику вероятности $P_{r}$.

Введем необходимые обозначения. Пусть

$$
\begin{gathered}
m=\mathbf{E} \xi_{1}, \quad m_{r}=\mathbf{E} \xi_{1}^{(r)} \\
\sigma=\sqrt{\mathbf{D} \xi_{1}}, \quad \sigma_{r}=\sqrt{\mathbf{D} \xi_{1}^{(r)}}, \quad \mu_{4}=\mathbf{E}\left(\xi_{1}-m\right)^{4} .
\end{gathered}
$$

Положим также

$$
\varphi(X ; t)=\mathbf{E} e^{i t X}
$$

для любой случайной величины $X$. Выделим несколько зон изменения параметров:

(a) $N \rightarrow \infty, n-N-$ постоянная величина,

(b) $N \rightarrow \infty, n-N \rightarrow \infty, n-N=o(N)$,

(c) $N \rightarrow \infty, n-N \asymp N$ (здесь и далее знак $\asymp$ означает, что связанные им величины имеют одинаковый порядок [5]),

(d) $N \rightarrow \infty, n / N \rightarrow \infty, N / \ln n \rightarrow \infty$,

(e) $N \rightarrow \infty, N / \ln n \rightarrow \alpha, \alpha>0$ - положительная постоянная,

(f) $N / \ln n \rightarrow 0$. 
Как видно из (2), распределения сумм $\zeta_{N}, \zeta_{N-l}^{(r)}+\tilde{\zeta}_{l}^{(r)}$ зависят от параметра $x$, который может быть выбран произвольно из интервала $(0,1)$. Мы будем выбирать его наиболее удобным для получения локальных теорем способом, а именно, так, чтобы математическое ожидание суммы $\zeta_{N}$ было близко к $n$. Для зоны (а) положим

$$
x=\frac{1}{N},
$$

для зон (b)-(d) выбираем $x$ так, чтобы удовлетворялось равенство

$$
m=\frac{x}{(1-x) \ln (1-x)^{-1}}=\frac{n}{N},
$$

а в зонах (e) и (f) положим

$$
x=1-\frac{1}{n} .
$$

Нетрудно проверить, что при таком выборе параметра $x$ в зоне (е)

$$
\mu_{4} /\left(\sigma^{4} N\right) \rightarrow \alpha^{-1},
$$

в то время как в зонах (a)-(d)

$$
\mu_{4}=o\left(\sigma^{4} N\right)
$$

В других аналогичных задачах разбиение на зоны тоже производится в зависимости от поведения дробей $n / N$ и $\mu_{4} /\left(\sigma^{4} N\right)$ (см., например, [6, 7]).

Говорят [8], что в обобщенной схеме размещения (1) возникает гигантская компонента, если при $n \rightarrow \infty$ случайная величина $\eta_{(N)}$ с вероятностью, стремящейся к 1 , имеет порядок $n$, и случайные величины $\eta_{(N)}$ и $\eta_{(N-1)}$ имеют предельные распределения при различной по порядку нормировке. Как показывают исследования $[6,7,8]$, именно при условии

$$
\sigma^{4} N=O\left(\mu_{4}\right)
$$

то есть в последних двух зонах, следует ожидать появления гигантской компоненты.

Из результатов работы [4] ясно, что гигантский цикл возникает в зоне (f) и не возникает в зонах (a)-(d). Таким образом, остался открытым вопрос о его возникновении в зоне (e). Ниже будет показано, что в рассматриваемых случайных подстановках гигантская компонента возникает только в зоне (f).

Теоремы 1-6 содержат результаты о предельном поведении $\eta_{(N-p)}$ при фиксированном $p$ в зонах (a)-(f).

Теорема 1. Пусть $N, n \rightarrow \infty$ так, что $n-N-$ постоянная величина. Тогда для $p=0,1, \ldots, n-N-1$

$$
\mathbf{P}\left\{\eta_{(N-p)}=2\right\} \rightarrow 1
$$

Теорема 2. Пусть $N, n \rightarrow \infty$ так, что

$$
n-N \rightarrow \infty, \quad n-N=o(N),
$$


параметр х выбран как корень уравнения (4), а г удовлетворяет соотношениям

$$
\begin{aligned}
N x^{r-1} / r & \rightarrow \infty, \\
N x^{r} /(r+1) & \rightarrow \gamma,
\end{aligned}
$$

где $\gamma$ - неотричательная постоянная.

Тогда для любого фиксированного $p=0,1,2, \ldots$

$$
\begin{gathered}
\mathbf{P}\left\{\eta_{(N-p)}=r\right\} \rightarrow e^{-\gamma} \sum_{l=0}^{p} \frac{\gamma^{l}}{l !}, \\
\mathbf{P}\left\{\eta_{(N-p)}=r+1\right\} \rightarrow 1-e^{-\gamma} \sum_{l=0}^{p} \frac{\gamma^{l}}{l !} .
\end{gathered}
$$

Теорема 3. Пусть $N, n \rightarrow \infty$ так, что

$$
n-N \asymp N
$$

параметр х выбран как корень уравнения (4), а r удовлетворяет соотночению

$$
n x^{r} / r \rightarrow \gamma
$$

где $\gamma$ - положительная постоянная. Тогда для любого фиксированного $p=0,1,2, \ldots u$ любого челого фиксированного $h$

$$
\mathbf{P}\left\{\eta_{(N-p)} \leqslant r+h\right\} \sim e^{-\gamma x^{h}} \sum_{l=0}^{p} \frac{\left(\gamma x^{h}\right)^{l}}{l !}
$$

Теорема 4. Пусть $N, n \rightarrow \infty$ так, что

$$
n / N \rightarrow \infty, \quad N / \ln n \rightarrow \infty
$$

параметр х выбран как корень уравнения (4), r удовлетворяет соотночению

$$
n x^{r} / r \rightarrow \gamma
$$

где $\gamma$ - положительная постоянная. Тогда для любого фиксированного $p=0,1,2, \ldots$

$$
\mathbf{P}\left\{\eta_{(N-p)} \leqslant r\right\} \rightarrow e^{-\gamma} \sum_{l=0}^{p} \frac{\gamma^{l}}{l !}
$$

Введем обозначения

$$
\begin{aligned}
& I_{0}(y, z)=y^{\alpha-1}, \\
& I_{s}(y, z)=\int_{X(s, y, z)} \frac{\left(y-x_{1}-\ldots-x_{s}\right)^{\alpha-1}}{x_{1} \ldots x_{s}} d x_{1} \ldots d x_{s},
\end{aligned}
$$

где

$$
X(s, y, z)=\left\{\left(x_{1}, \ldots, x_{s}\right): x_{k} \geqslant z, y \geqslant x_{1}+\cdots+x_{s}\right\}, \quad s=1,2, \ldots
$$


Теорема 5. Пусть $N, n \rightarrow \infty$ так, что

$$
N / \ln n \rightarrow \alpha,
$$

где $\alpha$ - положительная постоянная. Тогда для любого фиксированного $p=0,1,2, \ldots u$ любого фиксированного $z>0$

$$
\mathbf{P}\left\{\eta_{(N-p)} \leqslant z n\right\} \rightarrow \sum_{s=0}^{\infty} \frac{(-\alpha)^{s}}{s !} I_{s}(1, z)(-1)^{\min \{p, s\}}\left(\begin{array}{c}
s-1 \\
\min \{p, s\}
\end{array}\right) .
$$

Теорема 6. Пусть $n \rightarrow \infty$ так, что

$$
N / \ln n \rightarrow 0
$$

г удовлетворяет соотночению

$$
\ln (n / r) \sim \gamma(\ln n) / N
$$

гое $\gamma$ - положительная постоянная. Тогда для любого фиксированного $p=1,2, \ldots$

$$
\mathbf{P}\left\{\eta_{(N-p)} \leqslant r\right\} \rightarrow e^{-\gamma} \sum_{l=0}^{p-1} \frac{\gamma^{l}}{l !}
$$

если $N \rightarrow \infty, u$

$$
\mathbf{P}\left\{\eta_{(N-p)} \leqslant r\right\} \rightarrow \sum_{l=0}^{p-1}\left(\begin{array}{c}
N-1 \\
l
\end{array}\right) \frac{\gamma^{l}}{N^{l}}\left(1-\frac{\gamma}{N}\right)^{N-l-1},
$$

если $N$ фиксировано и $\gamma<N$.

В [4] показано, что в зоне (а) имеет место соотношение

$$
\mathbf{P}\left\{\eta_{(N)}=2\right\} \rightarrow 1
$$

Отсюда легко видеть, что и для $p=1, \ldots, n-N-1$ справедливо соотношение

$$
\mathbf{P}\left\{\eta_{(N-p)}=2\right\} \rightarrow 1
$$

Тем самым, теорема 1 доказана.

Рассмотрим зоны (a)-(d). По теоремам 4.2.9, 4.2.7, 4.2.5 из [1] и лемме 15 работы [4] при условии, что $N P_{r} \rightarrow \gamma$, где $\gamma$ - положительная постоянная, получаем, что

$$
\begin{aligned}
\mathbf{P}\left\{\zeta_{N}=k\right\} & =\frac{1+o(1)}{\sigma \sqrt{2 \pi N}} e^{-u^{2} / 2} \\
\mathbf{P}\left\{\zeta_{N}^{(r)}=k\right\} & =\frac{1+o(1)}{\sigma_{r} \sqrt{2 \pi N}} e^{-u_{r}^{2} / 2}
\end{aligned}
$$


равномерно относительно целых $k$, для которых

$$
\begin{aligned}
u & =\frac{k-n}{\sigma \sqrt{N}}, \\
u_{r} & =\frac{k-N m_{r}}{\sigma_{r} \sqrt{N}}
\end{aligned}
$$

лежат в произвольных фиксированных конечных интервалах. Второе из соотношений в (5) сохраняет силу в зоне (b) при $r \geqslant 2$, если $N P_{r-1} \rightarrow \infty, N P_{r} \rightarrow \gamma$, где постоянная $\gamma \geqslant 0$. Кроме того, в этом случае указанное соотношение сохраняет силу для суммы $\zeta_{N}^{(r+1)}$, а при $r \geqslant 3$ и для $\zeta_{N}^{(r-1)}$.

Из лемм 3-5 работы [4] следует, что если $r$ в зонах (b)-(d) выбрано так, как указано в условиях теорем 2-4, то $N P_{r} \rightarrow \gamma$. Таким образом, в условиях теорем 2-4 справедливы соотношения (5). Кроме того, с помощью найденных в [4] соотношений легко установить, что $\sigma_{r} \sim \sigma, m_{r} \sim m$.

Найдем асимптотику $\zeta_{N-l}^{(r)}+\tilde{\zeta}_{l}^{(r)}$ при любом фиксированном $l$.

Лемма 1. В условиях теорем 2-4 при любых фиксированных $t$ и l выполняется соотношение

$$
\varphi\left(\frac{\zeta_{N-l}^{(r)}+\tilde{\zeta}_{l}^{(r)}-N m_{r}}{\sigma_{r} \sqrt{N}} ; t\right) \rightarrow e^{-t^{2} / 2}
$$

Доказательство. Нетрудно видеть, что

$$
\varphi\left(\frac{\zeta_{N-l}^{(r)}+\tilde{\zeta}_{l}^{(r)}-N m_{r}}{\sigma_{r} \sqrt{N}} ; t\right)=\varphi\left(\frac{\zeta_{N}^{(r)}-N m_{r}}{\sigma_{r} \sqrt{N}} ; t\right) \frac{\varphi\left(\tilde{\zeta}_{l}^{(r)} ; t /\left(\sigma_{r} \sqrt{N}\right)\right)}{\varphi\left(\zeta_{l}^{(r)} ; t /\left(\sigma_{r} \sqrt{N}\right)\right)}
$$

Пользуясь найденными в [4] оценками моментов $\xi_{1}^{(r)}$, легко показать, что $m_{r} /\left(\sigma_{r} \sqrt{N}\right) \rightarrow 0$. Отсюда и из представления $\varphi\left(\xi_{1}^{(r)} ; u\right)=1+O\left(m_{r} u\right)$ следует, что $\varphi\left(\zeta_{l}^{(r)} ; t /\left(\sigma_{r} \sqrt{N}\right)\right) \rightarrow 1$

Нетрудно показать также, что $\mathbf{E} \tilde{\xi}_{1}^{(r)} \asymp r$. Действительно,

$$
\mathbf{E} \tilde{\xi}_{1}^{(r)}=\frac{\sum_{k=r+1}^{\infty} x^{k}}{\sum_{k=r+1}^{\infty} x^{k} / k}=\frac{x^{r+1}}{P_{r}(1-x) \ln (1-x)^{-1}}=\frac{x^{r} m}{P_{r}}=\frac{n x^{r}}{N P_{r}} .
$$

В зоне (b) $n \sim N$ и в силу формулы (7) в [4]

$$
N P_{r} \sim \frac{N x^{r}}{r+1}
$$

откуда получаем, что $\mathbf{E} \tilde{\xi}_{1}^{(r)} \sim r+1$. Поскольку в условиях теоремы 3 справедливы соотношения $n \asymp N$ и $N x^{r} \asymp r$, при $N P_{r} \rightarrow \gamma>0$ получим, что $\mathbf{E} \tilde{\xi}_{1}^{(r)} \asymp r$. В условиях теоремы 4 справедливы соотношения $n x^{r} / r \rightarrow \gamma$ и $N P_{r} \rightarrow \gamma$, откуда получаем, что $\mathbf{E} \tilde{\xi}_{1}^{(r)} \sim r$.

Докажем, что $r=o(\sigma \sqrt{N})$. Для этого оценим $N P_{\varepsilon \sigma \sqrt{N}}$, где $\varepsilon$ - положительная постоянная. Поскольку $m=o(\sigma \sqrt{N})$,

$$
N P_{\varepsilon \sigma \sqrt{N}} \leqslant N \mathbf{P}\left\{\left|\xi_{1}-m\right| \geqslant \varepsilon \sigma \sqrt{N} / 2\right\}=O\left(\mu_{4} /\left(\sigma^{4} N\right)\right)
$$


Как уже отмечалось выше, в зонах (b)-(d) $\mu_{4}=o\left(\sigma^{4} N\right)$. Поэтому $N P_{\varepsilon \sigma \sqrt{N}} \rightarrow 0$ при любом $\varepsilon>0$. В то же время $N P_{r} \rightarrow \gamma$, что, как было отмечено ранее, вытекает из условий теорем 2-4. Следовательно, $r=o(\sigma \sqrt{N})$ при $\gamma>0$. При $\gamma=0$ воспользуемся следующим условием $N P_{r-1} \rightarrow \infty$ теоремы 2. Отсюда следует, что $r-1=o(\sigma \sqrt{N})$, а значит, $r=o(\sigma \sqrt{N})$.

Отсюда и из представления

$$
\varphi\left(\tilde{\xi}_{1}^{(r)} ; t /\left(\sigma_{r} \sqrt{N}\right)\right)=1+O\left(\mathbf{E} \tilde{\xi}_{1}^{(r)} /\left(\sigma_{r} \sqrt{N}\right)\right)
$$

с помощью соотношения $\sigma_{r} \sim \sigma$ находим, что

$$
\varphi\left(\tilde{\zeta}_{l}^{(r)} ; t /\left(\sigma_{r} \sqrt{N}\right)\right) \rightarrow 1
$$

Теперь, используя (5), получаем утверждение леммы.

Локальную сходимость $\zeta_{N-l}^{(r)}+\tilde{\zeta}_{l}^{(r)}$ к нормальному закону легко доказать, следуя доказательству леммы 15 в [4] и используя оценку

$$
\left|\varphi\left(\zeta_{N-l}^{(r)}+\tilde{\zeta}_{l}^{(r)} ; u\right)\right| \leqslant\left|\varphi\left(\zeta_{N-l}^{(r)} ; u\right)\right|
$$

справедливую, поскольку величина $N-l$ удовлетворяет тем же условиям, что и величина $N$. Итак, справедливо следующее утверждение.

Лемма 2. В условиях теорем 2-4 для любого фиксированного $l=0,1,2, \ldots$

$$
\mathbf{P}\left\{\zeta_{N-l}^{(r)}+\tilde{\zeta}_{l}^{(r)}=k\right\}=\frac{1}{\sigma_{r} \sqrt{2 \pi N}} e^{-u_{r}^{2} / 2}(1+o(1))
$$

равномерно по всем $k$ таким, что и лежит в любом конечном фиксированном интервале.

Теперь, используя соотношения (5) и (6) при $k=n$, учитывая, что согласно [4] $\left(n-N m_{r}\right)^{2}=o\left(N \sigma_{r}^{2}\right)$ и $\sigma \sim \sigma_{r}$, и используя формулу (3), получаем, что

$$
\mathbf{P}\left\{\eta_{(N-p)} \leqslant r\right\} \sim \sum_{l=0}^{p}\left(\begin{array}{l}
N \\
l
\end{array}\right) P_{r}^{l}\left(1-P_{r}\right)^{N-l}
$$

Далее, при $N \rightarrow \infty$ и фиксированном $l$

$$
\left(\begin{array}{l}
N \\
l
\end{array}\right)=\frac{N !}{(N-l) ! l !} \sim \frac{N^{l}}{l !} .
$$

Поскольку в условиях теоремы $2 x \rightarrow 0$, кроме соотношения $N P_{r} \rightarrow \gamma$ также справедливы соотношения $N P_{r-1} \rightarrow \infty$ и $N P_{r+1} \rightarrow 0$. Отсюда легко найти, что в условиях теоремы 2

$$
\begin{aligned}
\mathbf{P}\left\{\eta_{(N-p)} \leqslant r-1\right\} & \rightarrow 0, \\
\mathbf{P}\left\{\eta_{(N-p)} \leqslant r\right\} & \rightarrow e^{-\gamma} \sum_{l=0}^{p} \gamma^{l} / l !, \\
\mathbf{P}\left\{\eta_{(N-p)} \leqslant r+1\right\} & \rightarrow 1,
\end{aligned}
$$


откуда следует утверждение теоремы 2.

В условиях теоремы $3 N P_{r+h} \sim \gamma x^{h}$ при любом целом фиксированном $h$, что доказано в [4]. Отсюда следует утверждение теоремы 3.

Наконец, теорема 4 следует из соотношений (7), (8) и $N P_{r} \rightarrow \gamma$.

Рассмотрим зону (е). Полагая $x=1-1 / n, r=z n$, где постоянная $z \in(0 ; 1)$, и применяя лемму 6 из [4], находим, что $N P_{r} \rightarrow E(z, 0)$, где

$$
E(z, t)=\alpha \int_{z}^{\infty} y^{-1} e^{-y(1-i t)} d y .
$$

Далее, по теореме 4.2.1 из [1] распределение случайной величины $\zeta_{N} / n$ сходится к гаммарагпределению с параметрами $(\alpha, 1)$, то есть для целых $y n$

$$
n \mathbf{P}\left\{\zeta_{N}=y n\right\} \rightarrow \frac{y^{\alpha-1} e^{-y}}{\Gamma(\alpha)}
$$

равномерно по $y$ в любом положительном конечном фиксированном интервале.

Найдем предельное распределение $\zeta_{N-l}^{(r)}+\tilde{\zeta}_{l}^{(r)}$.

Лемма 3. Пусть $r=z n$, величина $z \in(0 ; 1), x=1-n^{-1}$. Тогда в зоне (е) при любых фиксированных $t$ ul

$$
\varphi\left(\zeta_{N-l}^{(r)}+\tilde{\zeta}_{l}^{(r)} ; t / n\right) \rightarrow \frac{\exp \{E(z, 0)-E(z, t)\} E(z, t)^{l}}{(1-i t)^{\alpha} E(z, 0)^{l}}
$$

Доказательство. Легко видеть, что

$$
\varphi\left(\zeta_{N-p}^{(r)}+\tilde{\zeta}_{p}^{(r)}: t / n\right)=\varphi\left(\zeta_{N}^{(r)} ; t / n\right) \frac{\varphi\left(\tilde{\zeta}_{p}^{(r)} ; t / n\right)}{\varphi\left(\zeta_{p}^{(r)} ; t / n\right)} .
$$

Используя оценки моментов $\xi_{1}^{(r)}$, полученные в [4], нетрудно показать, что $m_{r}=o(n)$. Отсюда следует, что $\varphi\left(\zeta_{l}^{(r)} ; t / n\right) \rightarrow 1$.

При доказательстве леммы 1.8 .8 в [2] показано, что

$$
\sum_{s=r+1}^{\infty} s^{-1} x^{s} e^{i t s / n} \rightarrow E(z, t) / \alpha
$$

Отсюда получаем, что

$$
\varphi\left(\tilde{\xi}_{1}^{(r)} ; t / n\right)=\sum_{s=r+1}^{\infty} \frac{x^{s} e^{i t s / n}}{s P_{r} \ln (1-x)^{-1}} \sim \frac{E(z, t)}{P_{r} \alpha \ln n} \rightarrow \frac{E(z, t)}{E(z, 0)}
$$

где мы воспользовались условием $N \sim \alpha \ln n$ и тем, что $N P_{r} \rightarrow E(z, 0)$, как отмечалось выше.

В лемме 16 из [4] приведено асимптотическое выражение для $\varphi\left(\zeta_{N}^{(r)} ; t / n\right)$, равное $\exp \{E(z, 0)-E(z, t)\}(1-i t)^{-\alpha}$. Тем самым лемма доказана. 
Лемма 4. Пусть $r=z n$, величина $z \in(0 ; 1), x=1-n^{-1}$. Тогда в зоне (е) при любом фиксированном l для цельх уп

$$
n \mathbf{P}\left\{\zeta_{N-l}^{(r)}+\tilde{\zeta}_{l}^{(r)}=y n\right\} \rightarrow \frac{\alpha^{l} e^{E(z, 0)-y}}{\Gamma(\alpha) E(z, 0)^{l}} \sum_{s=0}^{\infty} \frac{(-\alpha)^{s}}{s !} I_{s+l}(y, z)
$$

равномерно по у в любом конечном фиксированном интервале положительной полуоси.

Доказательство. Как показано в [4], характеристической функции

$$
\exp \{E(z, 0)-E(z, t)\}(1-i t)^{-\alpha}
$$

соответствует плотность

$$
g(y)=\frac{e^{E(z .0)-y}}{\Gamma(\alpha)} \sum_{s=0}^{\infty} \frac{(-\alpha)^{s}}{s !} I_{s}(y, z), \quad y>0,
$$

а характеристической функции $E(z, t) / E(z, 0)$ соответствует плотность

$$
\alpha y^{-1} E(z, 0)^{-1} e^{-y}, \quad y>z .
$$

Пользуясь формулой свертки, нетрудно найти, что характеристической функции

$$
\frac{\exp \left\{E(z, 0)-E(z, t\} E(z, t)^{l}\right.}{(1-i t)^{\alpha} E(z, 0)^{l}}
$$

соответствует плотность

$$
G(y)=\frac{\alpha^{l} e^{E(z .0)-y}}{\Gamma(\alpha) E(z, 0)^{l}} \sum_{s=0}^{\infty} \frac{(-\alpha)^{s}}{s !} I_{s+l}(y, z), \quad y>0 .
$$

По формуле обращения находим, что

$$
2 \pi n \mathbf{P}\left\{\zeta_{N-l}^{(r)}+\tilde{\zeta}_{l}^{(r)}=y n\right\}-2 \pi n G(y)=I_{1}+I_{2}+I_{3}+I_{4}
$$

где

$$
\begin{aligned}
& I_{1}=\int_{-A}^{A} e^{-i t y}\left(\varphi\left(\zeta_{N-l}^{(r)}+\tilde{\zeta}_{l}^{(r)} ; t / n\right)-\frac{\exp \{E(z, 0)-E(z, t)\} E(z, t)^{l}}{(1-i t)^{\alpha} E(z, 0)^{l}}\right) d t, \\
& I_{2}=\int_{A<|t| \leqslant \varepsilon n} e^{-i t y} \varphi\left(\zeta_{N-l}^{(r)}+\tilde{\zeta}_{l}^{(r)} ; t / n\right) d t, \\
& I_{3}=\int_{\varepsilon n<|t| \leqslant \pi n} e^{-i t y} \varphi\left(\zeta_{N-l}^{(r)}+\tilde{\zeta}_{l}^{(r)} ; t / n\right) d t, \\
& I_{4}=-\int_{A<|t|} e^{-i t y} \frac{\exp \{E(z, 0)-E(z, t)\} E(z, t)^{l}}{(1-i t)^{\alpha} E(z, 0)^{l}} d t,
\end{aligned}
$$

где $A$ и $\varepsilon-$ некоторые положительные постоянные. Для доказательства леммы необходимо показать, что каждый из перечисленных интегралов может быть сделан сколь угодно малым выбором достаточно больших $N, n, A$ и достаточно малого $\varepsilon$. 
По лемме 3 первый интеграл стремится к нулю при любом фиксированном $A$. Для оценки интегралов $I_{2}$ и $I_{3}$ заметим, что

$$
\left|\varphi\left(\zeta_{N-p}^{(r)}+\tilde{\zeta}_{p}^{(r)} ; t / n\right)\right| \leqslant\left|\varphi\left(\zeta_{N-p}^{(r)} ; t / n\right)\right| .
$$

Величина $N-l$ удовлетворяет тем же условиям, что и величина $N$, поэтому здесь применимы те же рассуждения, что и в лемме 17 в [4], позволяющие свести оценку интегралов $I_{2}, I_{3}$ к оценкам одноименных интегралов, найденным при доказательстве теоремы 4.2.1 в [1].

Для оценки $I_{4}$ проведем интегрирование по частям. Учитывая, что

$$
\frac{\partial}{\partial t} E(z, t)=\frac{i \alpha}{1-i t} e^{z(i t-1)}
$$

находим, что

$$
\begin{gathered}
\int_{A}^{\infty} e^{-i t y} \frac{\exp \{E(z, 0)-E(z, t)\} E(z, t)^{l}}{(1-i t)^{\alpha} E(z, 0)^{l}} d t=\frac{e^{E(z, 0)}}{E(z, 0)^{l}}\left(-\left.\frac{e^{-i t y} e^{-E(z, t)} E(z, t)^{l}}{i y(1-i t)^{\alpha}}\right|_{A} ^{\infty}\right. \\
\left.+i \alpha \int_{A}^{\infty} e^{-i t y-E(z, t)} E(z, t)^{l-1} \frac{(l-E(z, t)) e^{z(i t-1)}+E(z, t)}{(1-i t)^{\alpha+1}} d t\right) .
\end{gathered}
$$

Отсюда видно, что

$$
\left|I_{4}\right| \leqslant \frac{c}{y}\left(\frac{1}{\left(1+A^{2}\right)^{\alpha / 2}}+\int_{A}^{\infty} \frac{d t}{\left(1+t^{2}\right)^{(\alpha+1) / 2}}\right),
$$

где $c$ - некоторая положительная постоянная. Поэтому интеграл $I_{4}$ может быть сделан сколь угодно малым выбором достаточно большого $A$. Лемма доказана.

Теперь, из формулы (3), полагая $r=z n$ и используя (9) и (10) при $y=1$, а также соотношение (8), получаем, что

$$
\begin{aligned}
\mathbf{P}\left\{\eta_{(N-p)} \leqslant z n\right\} & \sim \sum_{l=0}^{p}\left(\begin{array}{c}
N \\
l
\end{array}\right) P_{r}^{l}\left(1-P_{r}\right)^{N-l} \frac{\alpha^{l} e^{E(z, 0)}}{E(z, 0)^{l}} \sum_{s=0}^{\infty} \frac{(-\alpha)^{s}}{s !} I_{s+l}(1, z) \\
& \sim \sum_{l=0}^{p} \frac{\left(\alpha N P_{r}\right)^{l} e^{-N P_{r}+E(z, 0)}}{l ! E(z, 0)^{l}} \sum_{s=l}^{\infty} \frac{(-\alpha)^{s-l} s !}{(s-l) ! s !} I_{s}(1, z) \\
& \rightarrow \sum_{s=0}^{\infty} \frac{(-\alpha)^{s}}{s !} I_{s}(1, z) \sum_{l=0}^{\min (p, s\}}(-1)^{l}\left(\begin{array}{l}
s \\
l
\end{array}\right) .
\end{aligned}
$$

Последнее выражение можно упростить по формуле (5.114) из [5] и получить утверждение теоремы 5.

Рассмотрим зону (f).

Лемма 5. Пусть выполнены условия зоны (f), $x=1-n^{-1}, r$ выбрано так, что

$$
\ln (n / r) \sim \gamma(\ln n) / N
$$


где $\gamma$ - положительная постоянная. Тогда для любого челого положительного $l$

$$
\mathbf{P}\left\{\tilde{\zeta}_{l}^{(r)}=h\right\}=\frac{N l e^{-h / n}}{h \gamma \ln n}(1+o(1))
$$

равномерно по челым $h$ таким, что $0<c_{1} \leqslant h / n \leqslant c_{2}<\infty$, где $c_{1}, c_{2}-$ некоторые постоянные.

Доказательство. Докажем это утверждение по индукции. Пусть $l=1$. Заменяя суммирование интегрированием, нетрудно получить, что

$$
\sum_{k=r+1}^{\infty} \frac{x^{k}}{k} \sim \int_{r(1-x)}^{\infty} e^{-u} u^{-1} d u \sim-\ln (r(1-x))=\ln \left(\frac{n}{r}\right) \sim \gamma \frac{\ln n}{N}
$$

Поэтому

$$
\mathbf{P}\left\{\tilde{\xi}_{1}^{(r)}=h\right\}=\frac{x^{h}}{h \sum_{k=r+1}^{\infty} x^{k} / k} \sim \frac{N e^{-h / n}}{h \gamma \ln n} .
$$

Предположим, что утверждение леммы справедливо для $l=L-1$, докажем его для $l=L$. Пусть $\varepsilon \in(0 ; 1)$. Тогда

$$
\begin{aligned}
\mathbf{P}\left\{\tilde{\zeta}_{L}^{(r)}=h\right\}= & \sum_{0<j<\varepsilon h} \mathbf{P}\left\{\tilde{\zeta}_{L-1}^{(r)}=j\right\} \mathbf{P}\left\{\tilde{\xi}_{1}^{(r)}=h-j\right\} \\
& +\sum_{\varepsilon h \leqslant j \leqslant h} \mathbf{P}\left\{\tilde{\zeta}_{L-1}^{(r)}=j\right\} \mathbf{P}\left\{\tilde{\xi}_{1}^{(r)}=h-j\right\} .
\end{aligned}
$$

Рассмотрим первую сумму. Легко видеть, что

$$
\mathbf{P}\left\{\tilde{\xi}_{1}^{(r)}=h-j\right\} \sim \frac{N e^{(j-h) / n}}{(h-j) \gamma \ln n} .
$$

Отсюда следует, что равномерно по $j \in(0 ; \varepsilon h)$ величина

$$
N^{-1} e^{h / n} h \gamma(\ln n) \mathbf{P}\left\{\tilde{\xi}_{1}^{(r)}=h-j\right\}
$$

может быть сделана сколь угодно близкой к 1 выбором достаточно малого $\varepsilon$. Следовательно,

$$
\frac{e^{h / n} h \gamma \ln n}{N \mathbf{P}\left\{\tilde{\zeta}_{L-1}^{(r)}<\varepsilon h\right\}} \sum_{0<j<\varepsilon h} \mathbf{P}\left\{\tilde{\zeta}_{L-1}^{(r)}=j\right\} \mathbf{P}\left\{\tilde{\xi}_{1}^{(r)}=h-j\right\}
$$

может быть сделано сколь угодно близким к 1 выбором достаточно малого $\varepsilon$.

Ясно, что

$$
\mathbf{P}\left\{\tilde{\zeta}_{L-1}^{(r)}<\varepsilon h\right\}=1-\mathbf{P}\left\{\tilde{\zeta}_{L-1}^{(r)} \geqslant \varepsilon h\right\} .
$$

Оценивая математическое ожидание $\tilde{\xi}_{1}^{(r)}$, находим, что

$$
\begin{aligned}
\mathbf{E} \tilde{\xi}_{1}^{(r)} & =\frac{\sum_{k=r+1}^{\infty} x^{k}}{\sum_{k=r+1}^{\infty} x^{k} / k} \sim \frac{x^{r+1}}{(1-x) \ln (n / r)} \\
& \sim \frac{N n}{\gamma \ln n},
\end{aligned}
$$


и применяя неравенство Чебышева, получаем оценку

$$
\mathbf{P}\left\{\tilde{\zeta}_{L-1}^{(r)} \geqslant \varepsilon h\right\} \leqslant \frac{(L-1) \mathbf{E} \tilde{\xi}_{1}^{(r)}}{\varepsilon h} \asymp \frac{N}{\ln n}=o(1) .
$$

Следовательно, $\mathbf{P}\left\{\tilde{\zeta}_{L-1}^{(r)}<\varepsilon h\right\} \rightarrow 1$ при любом фиксированном $\varepsilon$. Таким образом, выражение

$$
N^{-1} e^{h / n} h \gamma(\ln n) \sum_{0<j<\varepsilon h} \mathbf{P}\left\{\tilde{\zeta}_{L-1}^{(r)}=j\right\} \mathbf{P}\left\{\tilde{\xi}_{1}^{(r)}=h-j\right\}
$$

может быть сделано сколь угодно близким к 1 выбором достаточно малого $\varepsilon$ и достаточно большого $n$.

Оценим вторую сумму в (11). Для этого используем предположение индукции об асимптотике вероятности $\mathbf{P}\left\{\tilde{\zeta}_{L-1}^{(r)}=j\right\}$, поскольку $j \asymp n$. Тогда

$$
\begin{aligned}
\sum_{\varepsilon h \leqslant j \leqslant h} \mathbf{P}\left\{\tilde{\zeta}_{L-1}^{(r)}=j\right\} \mathbf{P}\left\{\tilde{\xi}_{1}^{(r)}=h-j\right\} & \sim \sum_{\varepsilon h \leqslant j<h-r} \frac{N(L-1) e^{-j / n}}{j \gamma \ln n} \frac{N e^{(j-h) / n}}{(h-j) \gamma \ln n} \\
& =\frac{N^{2}(L-1) e^{-h / n}}{\gamma^{2}(\ln n)^{2}} \sum_{\varepsilon h \leqslant j<h-r} \frac{1}{j(h-j)} \\
& =\frac{N^{2}(L-1) e^{-h / n}}{\gamma^{2}(\ln n)^{2}} \sum_{r<j \leqslant(1-\varepsilon) h} \frac{1}{j(h-j)} .
\end{aligned}
$$

Заменяя суммирование интегрированием, находим, что

$$
\begin{aligned}
\sum_{r<j \leqslant(1-\varepsilon) h} \frac{1}{j(h-j)} & \sim \int_{r}^{(1-\varepsilon) h} \frac{d y}{y(h-y)}=\frac{1}{h}\left(\ln \frac{1-\varepsilon}{\varepsilon}+\ln \left(\frac{h}{r}-1\right)\right) \\
& \sim \frac{1}{h} \ln \frac{n}{r} \sim \frac{\gamma \ln n}{h N} .
\end{aligned}
$$

Поэтому

$$
\sum_{\varepsilon n \leqslant j \leqslant h} \mathbf{P}\left\{\tilde{\zeta}_{L-1}^{(r)}=j\right\} \mathbf{P}\left\{\tilde{\xi}_{1}^{(r)}=h-j\right\} \sim \frac{N(L-1) e^{-h / n}}{h \gamma \ln n} .
$$

Используя найденные асимптотики обеих сумм из (11), получаем, что величина $N^{-1} e^{h / n} h \gamma(\ln n) \mathbf{P}\left\{\tilde{\zeta}_{L}^{(r)}=h\right\}$ может быть сделана сколь угодно близкой к $L$ выбором достаточно малого $\varepsilon$ и достаточно большого $n$. Отсюда следует утверждение леммы.

Лемма 6. Пусть выполнены условия зоны (f) $u N \rightarrow \infty, x=1-n^{-1}, r$ выбрано так, что

$$
\ln (n / r) \sim \gamma(\ln n) / N
$$

где $\gamma$ - положительная постоянная. Тогоа для любого фиксированного $l=1,2, \ldots$

$$
\mathbf{P}\left\{\zeta_{N-l}^{(r)}+\tilde{\zeta}_{l}^{(r)}=n\right\}=\frac{N l e^{-1}}{n \gamma \ln n}(1+o(1)) .
$$

Утверждение остается справеоливым и при фиксированном $N$, если $\gamma<N$. 
Доказательство. Для любого $\varepsilon>0$ справедливо равенство

$$
\begin{aligned}
\mathbf{P}\left\{\zeta_{N-l}^{(r)}+\tilde{\zeta}_{l}^{(r)}=n\right\}= & \sum_{0<j<\varepsilon n} \mathbf{P}\left\{\zeta_{N-l}^{(r)}=j\right\} \mathbf{P}\left\{\tilde{\zeta}_{l}^{(r)}=n-j\right\} \\
& +\sum_{\varepsilon n \leqslant j \leqslant n} \mathbf{P}\left\{\zeta_{N-l}^{(r)}=j\right\} \mathbf{P}\left\{\tilde{\zeta}_{l}^{(r)}=n-j\right\}
\end{aligned}
$$

Рассмотрим первую сумму в этом равенстве. Поскольку $n-j \asymp n$ равномерно по $j \in(0 ; \varepsilon n)$, используя лемму 5 , получаем, что

$$
\mathbf{P}\left\{\tilde{\zeta}_{l}^{(r)}=n-j\right\}=\frac{N l e^{(j-n) / n}}{(n-j) \gamma \ln n}(1+o(1)) .
$$

Отсюда следует, что величина

$$
(N l)^{-1} \operatorname{e\gamma n}(\ln n) \mathbf{P}\left\{\tilde{\zeta}_{l}^{(r)}=n-j\right\}
$$

может быть сделана сколь угодно близкой к 1 выбором достаточно малого $\varepsilon$ и достаточно большого $n$ равномерно по $j \in(0 ; \varepsilon n)$. Используя неравенство Чебышева, нетрудно показать, что

$$
\mathbf{P}\left\{\zeta_{N-l}^{(r)} \geqslant \varepsilon n\right\} \rightarrow 0
$$

Действительно,

$$
m_{r}=\sum_{k=1}^{r} x^{k} / \sum_{k=1}^{r} x^{k} / k .
$$

Из соотношений $N / \ln n \rightarrow 0$ и $\ln (n / r) \sim \gamma(\ln n) / N$ следует, как легко видеть, что $r=o(n)$, откуда следует, что $x^{k} \rightarrow 1$ при $k=\{1, \ldots, r\}$. В то же время $\ln n-\ln r=o(\ln n)$ при $N \rightarrow \infty$, а при фиксированном $N$ справедливо соотношение $(\ln r) / \ln n \sim 1-\gamma / N$. В обоих случаях получаем, что $\ln r \asymp \ln n$. Таким образом,

$$
m_{r} \sim \frac{r}{\ln r} \asymp \frac{r}{\ln n}=o(n) .
$$

Отсюда следует, что $\mathbf{P}\left\{\zeta_{N-l}^{(r)} \geqslant \varepsilon n\right\} \rightarrow 0$ или $\mathbf{P}\left\{\zeta_{N-l}^{(r)}<\varepsilon n\right\} \rightarrow 1$. Используя найденную оценку выражения (13), получаем, что величина

$$
(N l)^{-1} \operatorname{e\gamma n}(\ln n) \sum_{0<j<\varepsilon n} \mathbf{P}\left\{\zeta_{N-l}^{(r)}=j\right\} \mathbf{P}\left\{\tilde{\zeta}_{l}^{(r)}=n-j\right\}
$$

может быть сделана сколь угодно близкой к 1 выбором достаточно малого $\varepsilon$ и достаточно большого $n$.

Перейдем к оценке второй суммы. Для этого воспользуемся тем, что, как следует из результатов статьи [4], $\mathbf{P}\left\{\eta_{(N)} \leqslant r\right\} \rightarrow 0$ при $r=o(n)$. Отсюда и из (3) следует, что

$$
\left(1-P_{r}\right)^{N} \mathbf{P}\left\{\zeta_{N}^{(r)}=n\right\} / \mathbf{P}\left\{\zeta_{N}=n\right\} \rightarrow 0 .
$$

Нетрудно показать, что при выбранных $x$ и $r$ справедливо соотношение $N P_{r} \rightarrow \gamma$, поскольку

$$
P_{r}=\sum_{k=r+1}^{\infty} \frac{x^{k}}{k \ln n} \sim \frac{\ln (n / r)}{\ln n} \sim \frac{\gamma}{N}
$$


Поэтому

$$
\mathbf{P}\left\{\zeta_{N}^{(r)}=n\right\}=o\left(\mathbf{P}\left\{\zeta_{N}=n\right\}\right) .
$$

Заметим, что условия зоны (f) позволяют вместо $n$ взять величину $\varepsilon n$, а вместо $N$ взять $N-l$. Приведенное выше соотношение при этом останется в силе. Таким образом,

$$
\mathbf{P}\left\{\zeta_{N-l}^{(r)}=j\right\}=o\left(\mathbf{P}\left\{\zeta_{N}=n\right\}\right)
$$

при $\varepsilon n \leqslant j \leqslant n$. Отсюда следует, что вторая сумма в (12) бесконечно мала в сравнении с $\mathbf{P}\left\{\zeta_{N}=n\right\}$. Из леммы 12 статьи [4] следует, что

$$
\mathbf{P}\left\{\zeta_{N}=n\right\} \sim N e^{-1} /(n \ln n) .
$$

Поэтому вторая сумма в (12) бесконечно мала по сравнению с первой.

Отсюда получаем, что величина

$$
(N l)^{-1} \operatorname{e\gamma n}(\ln n) \mathbf{P}\left\{\zeta_{N-l}^{(r)}+\tilde{\zeta}_{l}^{(r)}=n\right\}
$$

может быть сделана сколь угодно близкой к 1 выбором достаточно малого $\varepsilon$ и достаточно большого $n$, откуда следует утверждение леммы.

Из доказанной леммы, используя упомянутые в ней соотношения

$$
\begin{aligned}
N P_{r} & \rightarrow \gamma, \\
\mathbf{P}\left\{\zeta_{N}=n\right\} & \sim\left(N e^{-1}\right) /(n \ln n),
\end{aligned}
$$

а также формулу (3), соотношение (8) и то, что при выбранном $r$

$$
\mathbf{P}\left\{\eta_{(N)} \leqslant r\right\} \rightarrow 0,
$$

получаем утверждение теоремы 6.

В зонах (b)-(d), как мы видели при доказательстве леммы 1, имеет место соотношение

$$
r=o(\sigma \sqrt{N})
$$

при выбранном $r$. Кроме того, в [7] показано, что если для суммы $\zeta_{N}$ выполняется интегральная сходимость к нормальному закону, то

$$
\sigma \sqrt{N}=o(N m) .
$$

Поскольку в зонах (b)-(d) $n=N m$, получаем, что

$$
r=o(n) .
$$

В зоне (е), как видно из теоремы 5, наибольшие циклы имеют порядок $n$.

Таким образом, в зонах (a)-(e) гигантский цикл не возникает. Напротив, в зоне (f), как видно из теоремы 6, второй по величине цикл имеет порядок $o(n)$. В статье [4] доказано, чTо

$$
\eta_{(N)} \sim n
$$

в этой зоне. Таким образом, мы можем утверждать, что гигантский цикл возникает только в зоне (f). 


\section{Список литературы}

1. Колчин В. Ф., Случайпые графы, Наука, Москва, 2000.

2. Колчин В. Ф., Случайлые отображения. Наука, Москва, 1984.

3. Колчин В. Ф., Севастьянов Б. А., Чистяков В. П., Случайиые размещения. Наука, Москва, 1976.

4. Павлов Ю. Л., Лосева Е. А., Предельные распределения максимального объема дерева в случайном рекурсивном лесе. Дискретиая математика (2002) 14, №1, 60-74.

5. Грэхем Р., Кнут Д., Паташник О., Колкретиая математика. Мир, Москва, 1998.

6. Чеплюкова И. А., Возникновение гигантского дерева в случайном лесе. Дискретиая математика (1998) 10, №1, 111-126.

7. Казимиров Н. И., О некоторых условиях отсутствия гигантской компоненты в обобщенной схеме размещения. Дискретиая математика (2002) 14, №2, 107-118.

8. Колчин В. Ф., О существовании гигантской компоненты в схемах размещения частиц. Обозрение прикладной и промышлениой математики (2000) 7, №1, 112-113.

Статья поступила 08.04.2003. 